\title{
Design Optimization of a Hybrid Trapped Field Magnet Lens (HTFML)
}

\author{
Sora Namba, Hiroyuki Fujishiro, Mark D. Ainslie, Senior Member, IEEE, Keita Takahashi, Devendra K. Namburi, \\ Difan Zhou, and Tomoyuki Naito
}

\begin{abstract}
The concept of a hybrid trapped field magnet lens (HTFML) was recently proposed by the authors, which consists of a trapped field magnet (TFM) cylinder exploiting the "vortex pinning effect," combined with a superconducting bulk magnetic lens exploiting the "diamagnetic shielding effect." This HTFML can generate, within its bore, a magnetic field higher than the applied magnetic field, even after external field decreases to zero. In this paper, a design optimization of the inner $\mathrm{GdBaCuO}$ magnetic lens within the GdBaCuO TFM cylinder was carried out using numerical simulations based on the finite element method, in order to maximize the concentrated magnetic field. The HTFML with an optimized shape and size achieved a concentrated magnetic field of $B_{c}=5.6$ and $12.8 \mathrm{~T}$ at the center of the lens for applied magnetic fields of $B$ app $=3$ and $10 \mathrm{~T}$, respectively. A maximum tensile stress of $+135 \mathrm{MPa}$ exists in the outer GdBaCuO TFM cylinder during the magnetizing process for $B_{\text {app }}=10 \mathrm{~T}$, which exceeds the fracture strength of the bulk. This result suggests that mechanical reinforcement is necessary to avoid mechanical fracture under such high magnetic field conditions.
\end{abstract}

Index Terms-Hybrid trapped field magnet lens (HTFML), mechanical stress, numerical simulation, REBaCuO bulk superconductor, trapped field magnets

\section{INTRODUCTION}

$\mathbf{R}$ $\mathrm{EBaCuO}$ (where $\mathrm{RE}$ is a rare earth element or $\mathrm{Y}$ ) superconducting bulks can trap a large amount of magnetic flux in pinning centers using the "vortex pinning effect," and can be used as strong trapped-field magnets (TFMs) capable of generating over $17 \mathrm{~T}[1,2]$, which is attractive for various practical applications. The performance of TFMs by field-cooled magnetization (FCM) is mostly determined by the critical current density, $J_{\mathrm{c}}(B, T)$, and the diameter of the bulk material. In addition, a magnetic lens has been also investigated using the "diamagnetic shielding effect" as a magnetic field amplification technique, in which the magnetic flux generated from an outer superconducting coil magnet is concentrated in the center of the magnetic lens [3].

This research is partially supported from JSPS KAKENHI Grant No. 15K04646. M. D. Ainslie would like to acknowledge financial support from an Engineering and Physical Sciences Research Council (EPSRC) Early Career Fellowship EP/P020313/1. All data are provided in full in the results section of this paper.

S. Namba, H. Fujishiro K. Takahashi and T. Naito are with the Department of Physical Science and Materials Engineering, Faculty of Science and Engineering, Iwate University, Morioka 020-8551, Japan (e-mail: g0318128@iwate-u.ac.jp, fujishiro@iwate-u.ac.jp).

M. D. Ainslie is with Bulk Superconductivity Group, Department of Engineering, University of Cambridge, Cambridge CB2 1PZ, UK (e-mail: mark.ainslie@eng.cam.ac.uk)
A concentrated field of $B_{\mathrm{c}}=12.42 \mathrm{~T}$ has been achieved at $20 \mathrm{~K}$ for a background field of $8 \mathrm{~T}$ using a $\mathrm{GdBaCuO}$ magnetic lens [4], and $B_{\mathrm{c}}=30.4 \mathrm{~T}$ has been realized at the center of such a lens in a higher background field of $28.3 \mathrm{~T}$ [5]. Although the magnetic lens is a promising device to concentrate high magnetic fields, continuous operation of the external magnetic field source is required to produce the higher concentrated magnetic field.

Recently, we proposed a new concept of a hybrid trapped field magnet lens (HTFML), which consists of a TFM cylinder exploiting the vortex pinning effect, combined with a bulk magnetic lens exploiting the diamagnetic shielding effect [6]. The HTFML can reliably generate a concentrated magnetic field in the center of the magnetic lens higher than the trapped field of the TFM and the applied magnetic field, even after the external magnetizing field decreases to zero.

In this paper, the optimization of the shape and size of the inner $\mathrm{GdBaCuO}$ magnetic lens in the HTFML is carried out using numerical simulations, which is inserted into an outer GdBaCuO TFM cylinder. The mechanical stress during the magnetization process, resulting from the combination of the current and magnetic field in the HTFML, is also analyzed.

\section{NUMERICAL SIMULATION FRAMEWORK}

In this paper, the electromagnetic model presented in our original paper describing the concept of the HTFML [6] is coupled with a mechanical model and is used to further evaluate the performance of the HTFML during the magnetizing process. Fig. 1 shows the cross-section of the three-dimensional (3D) numerical model of the HTFML. Assuming that the $10 \mathrm{~T}$ superconducting solenoid magnet (170 $\mathrm{mm}$ in outer diameter (O.D.), $120 \mathrm{~mm}$ in inner diameter (I.D.), and $200 \mathrm{~mm}$ in height (H)) located in Iwate University is used as the magnetizing coil, the HTFML is assembled in a room temperature bore $100 \mathrm{~mm}$ in diameter. The $\mathrm{GdBaCuO}$ magnetic lens is inserted inside the $\mathrm{GdBaCuO}$ TFM cylinder (60 $\mathrm{mm}$ in O.D., $40 \mathrm{~mm}$ in I.D., and $60 \mathrm{~mm}$ in H). The magnetic lens part has two slits $10^{\circ}$ wide along the $y$-direction, whose role is to allow the magnetic flux to intrude into and be concentrated at the center of the lens.

To optimize the shape and the size of the magnetic lens, five variable parameters (outer diameter, $O D$, inner diameter, $I D$, inner diameter 2, ID2, outer height, $\mathrm{OH}$, and inner height, $I H)$ are changed independently, which are indicated in Fig. 1. The detailed values of the size of each parameter 


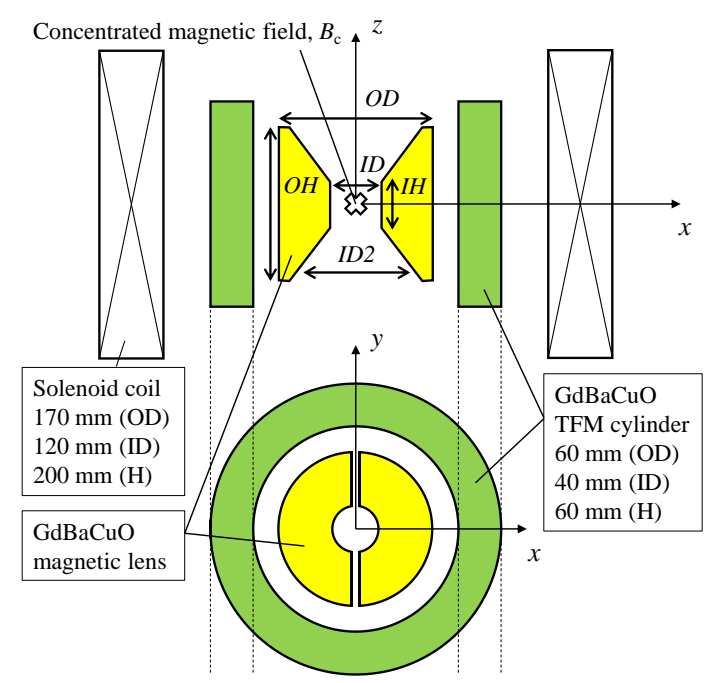

Fig. 1. Cross-section of three-dimensional numerical model of an HTFML in a magnetizing solenoid coil.

TABLE I

Five variable parameters of the $\mathrm{GdBaCuO}$ magnetic lens for the design

\begin{tabular}{cccccc}
\multicolumn{7}{c}{ optimization. } \\
\hline \hline Fig. No. & $\begin{array}{c}O D \\
(\mathrm{~mm})\end{array}$ & $\begin{array}{c}I D 2 \\
(\mathrm{~mm})\end{array}$ & $\begin{array}{c}I D \\
(\mathrm{~mm})\end{array}$ & $\begin{array}{c}O H \\
(\mathrm{~mm})\end{array}$ & $\begin{array}{c}I H \\
(\mathrm{~mm})\end{array}$ \\
\hline $3(\mathrm{a})$ & variable & variable & 10 & 45 & 10 \\
3 (b) & 36 & variable & 10 & 45 & 10 \\
3 (c) & 36 & 30 & 10 & variable & 10 \\
3 (d) & 36 & 30 & 10 & 30 & variable \\
\hline
\end{tabular}

$(O D \leq 36 \mathrm{~mm}, I D 2 \leq 36 \mathrm{~mm}, O H \leq 60 \mathrm{~mm})$ are shown in Table I, in which an $I D=10 \mathrm{~mm}$ is assumed for all optimization procedures to insert a Hall sensor for experiments planned in future work.

In the case that both magnetic lens and TFM cylinder are made of the same bulk superconducting material, it is necessary to control the temperature of each part independently. Fig. 2 shows the schematic view of the time step $(T S)$ dependence of the temperature, $T$, of the magnetic lens and the cylindrical TFM, and that of the external magnetic field, $B_{\text {ex }}$. The magnetizing procedure of the HTFML comprises the following four stages in this simulation.

1) The temperature of the outer $\mathrm{GdBaCuO}$ TFM cylinder is held at $T=100 \mathrm{~K}\left(>T_{\mathrm{c}}=92 \mathrm{~K}\right)$, while the $\mathrm{GdBaCuO}$ magnetic lens is cooled to $20 \mathrm{~K}$ (step 0 ).

2) The external magnetic field, $B_{\mathrm{ex}}$, is increased linearly up to the maximum magnetic field $B_{\text {app }}=3$ or $10 \mathrm{~T}$ at $+0.222 \mathrm{~T} / \mathrm{min}$ (steps 0-5). The magnetic lens is magnetized under zero-field cooling (ZFC) conditions at $20 \mathrm{~K}$, where the magnetic flux is concentrated in the bore of the lens.

3) The GdBaCuO TFM cylinder is then also cooled to $20 \mathrm{~K}$ $\left(<T_{\mathrm{c}}\right) \quad$ under $B_{\mathrm{app}}$, which transitions into the superconducting state (steps 5-6).

4) Finally, $B_{\mathrm{ex}}$ is ramped down from $B_{\text {app }}$ to zero at $-0.222 \mathrm{~T} / \mathrm{min}$ (steps 6-11). In this process, the $\mathrm{GdBaCuO}$ TFM cylinder is magnetized by FCM, which then acts like an external magnetizing coil magnet in a conventional magnetic lens setup, e.g., [3]. As a result, the magnetic flux

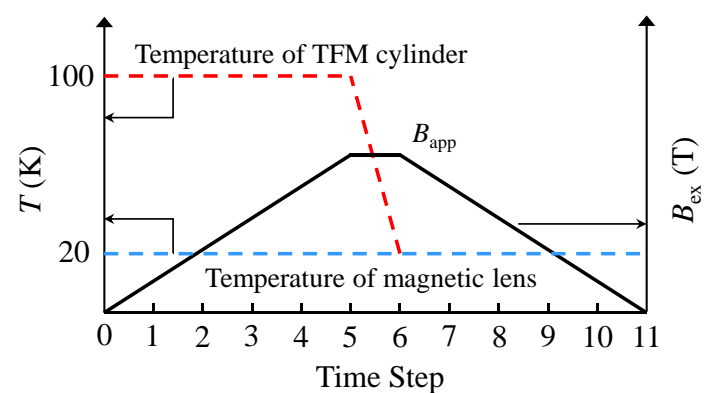

Fig. 2. Time step sequence of the temperature, $T$, of the $\mathrm{GdBaCuO}$ magnetic lens and $\mathrm{GdBaCuO} \mathrm{TFM}$ cylinder (left vertical axis), and the external magnetic field, $B_{\text {ex }}$ (right vertical axis).

generated from the TFM cylinder is concentrated in the magnetic lens and a magnetic field higher than $B_{\text {app }}$ is still present after the external $B_{\text {ex }}$ decreases to zero.

The $n$-power law $(n=20)$ is used to describe the nonlinear $E$ - $J$ characteristic of the bulk [7]. The $J_{\mathrm{c}}(B)$ characteristics at $20 \mathrm{~K}$ of the $\mathrm{GdBaCuO}$ bulk is defined by the following equation proposed by Jirsa et al. [8]

$$
J_{c}(B)=J_{c 1} \exp \left(-\frac{B}{B_{L}}\right)+J_{c 2} \frac{B}{B_{\max }} \exp \left[\frac{1}{k}\left(1-\left(\frac{B}{B_{\max }}\right)^{k}\right)\right]
$$

where $J_{\mathrm{c} 1}=9.0 \times 10^{9} \mathrm{~A} / \mathrm{m}^{2}, B_{\mathrm{L}}=1.5 \mathrm{~T}, J_{\mathrm{c} 2}=5.4 \times 10^{9} \mathrm{~A} / \mathrm{m}^{2}$, $B_{\max }=8.0 \mathrm{~T}$ and $k=0.5$ are used for the fitting parameters at $20 \mathrm{~K}[6,9]$.Elastic behavior can be expressed by Hooke's law, in which the stress tensor, $\sigma_{i j}$, is linearly proportional to strain tensor, $\varepsilon_{i j}$, as follows,

$$
\begin{gathered}
\sigma_{i j}=\lambda \cdot e_{k k} \cdot \delta_{i j}+2 G \cdot e_{i j}, \\
\lambda=\frac{v E}{(1-v)(1+2 v)} \\
G=\frac{E}{2(1+v)}
\end{gathered}
$$

where $\lambda$ and $G$ are the Lame's coefficients, $\delta_{i j}$ is the Kronecker delta. Young's modulus, $E=100 \mathrm{GPa}$, and Poisson ratio, $v=0.33$, are assumed as the mechanical parameters for the $\mathrm{REBaCuO}$ bulk [10]. The REBaCuO bulk is also assumed to be an isotropic and linear elastic material. The electromagnetic hoop stress, $\sigma_{\theta}$, is calculated for each time step of the magnetizing process.

\section{RESULTS AND DISCUSSION}

\section{A. Design optimization of the $\mathrm{GdBaCuO}$ magnetic lens}

Fig. 3 shows the concentrated magnetic field, $B_{\mathrm{c}}$, at the center of the magnetic lens $(x=y=z=0)$ for steps 5 and 11 for $B_{\text {app }}=3 \mathrm{~T}$, as a function of each variable. The magnetic field amplification factor, $B_{\mathrm{c}} / B_{\text {app }}$, is also shown on the right axis. In Fig. 3(a), the $B_{\mathrm{c}}$ vs $O D$ relationship is shown, in which the ID2 value is defined as $O D$ minus $6 \mathrm{~mm}$ $(I D 2=O D-6 \mathrm{~mm})$, because the ID2 changes depending on 

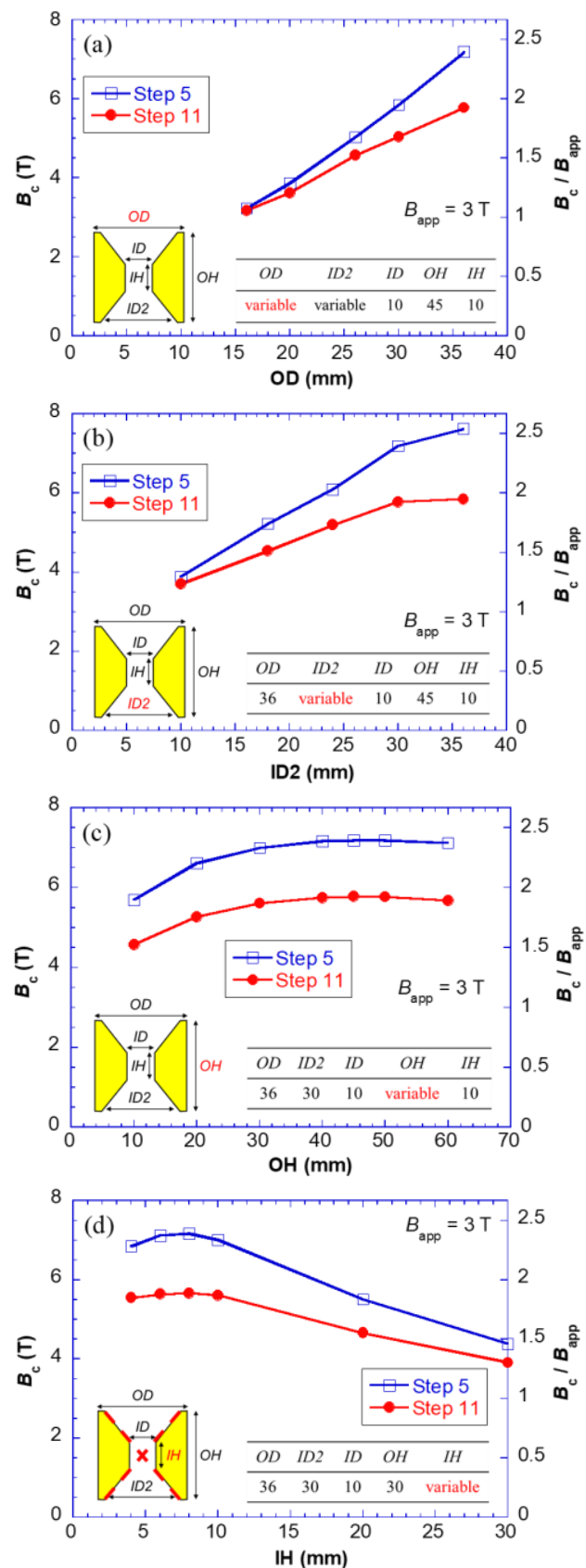

Fig. 3. The concentrated magnetic field, $B_{\mathrm{c}}$, at the center of the magnetic lens and the magnetic field amplification factor, $B_{\mathrm{c}} / B_{\text {app }}$, as a function of (a) $O D$, (b) $I D 2$, (c) $O H$, (d) $I H$ for $B_{\text {app }}=3 \mathrm{~T}$.

the $O D$ value. The $B_{\mathrm{c}}$ value increases linearly with increasing $O D$, and a $B_{\mathrm{c}} / B_{\text {app }}$ of 2.4 is achieved for $O D=36 \mathrm{~mm}$ at step 5, where only the magnetic lens is working and the magnetic flux produced by the magnetizing coil is efficiently concentrated in the bore of the lens. The $B_{\mathrm{c}}$ value at step 11, at which FCM of the outer TFM is finished, is lower than that at step 5, since the external magnetic field decreases during the descending stage and because of flux creep. However, a magnetic field higher than $B_{\text {app }}=3 \mathrm{~T}$ still remains for all cases of $O D$, which is the advantage of the HTFML.

Fig. 3(b) shows the ID2 dependence of $B_{\mathrm{c}}$ and $B_{\mathrm{c}} / B_{\text {app }}$ at steps 5 and 11 for $B_{\text {app }}=3 \mathrm{~T}$. For $I D 2=10 \mathrm{~mm}$, i.e., a cylindrical-shaped lens, which is a desirable shape from the viewpoint of machining the lens, the $B_{\mathrm{c}} / B_{\text {app }}$ is as small as 1.3. The $B_{\mathrm{c}}$ value increases with increasing ID2, similar to the $B_{\text {c }}$ vs $O D$ relationship shown in Fig. 3(a).

Fig. 3(c) shows the $O H$ dependence of $B_{\mathrm{c}}$ and $B_{\mathrm{c}} / B_{\text {app }}$ at steps 5 and 11 for $B_{\text {app }}=3 \mathrm{~T}$. With increasing $O H$, the $B_{\text {c value }}$ increases, takes a maximum for $\mathrm{OH}=45 \mathrm{~mm}$ and then decreases slightly for $O H=60 \mathrm{~mm}$, which is the same height as the outer $\mathrm{GdBaCuO}$ TFM cylinder. However, there is no significant difference between $O H=30-60 \mathrm{~mm}$. This result means that, in the present situation, $O H=30 \mathrm{~mm}$ is enough to maximize the $B_{\mathrm{c}}$ and $B_{\mathrm{c}} / B_{\text {app }}$ values and to provide a costeffective HTFML.

Fig. 3(d) shows the $I H$ dependence of $B_{\mathrm{c}}$ and $B_{\mathrm{c}} / B_{\text {app }}$ at steps 5 and 11 for $B_{\text {app }}=3 \mathrm{~T}$. For $I H=8 \mathrm{~mm}$, the highest $B_{\mathrm{c}}$ of $5.67 \mathrm{~T}$ is achieved at step 11 . The inset of Fig. 3(d) shows the cross-section of the magnetic lens for $I H=8 \mathrm{~mm}$, where the intersection of the lines extending from the lens taper corresponds to the lens center $(x=y=z=0)$. This result suggests that an appropriate taper angle for the magnetic lens can maximize the $B_{\mathrm{c}} / B_{\text {app }}$ value for a cylindrical TFM.

From the viewpoints of both the magnetic field amplification and cost-effectiveness, and based on these results, it was decided that the optimum shape of the $\mathrm{GdBaCuO}$ magnetic lens, which is inserted in the $\mathrm{GdBaCuO}$ TFM cylinder $(60 \mathrm{~mm}$ in O.D., $40 \mathrm{~mm}$ in I.D. and $60 \mathrm{~mm}$ in $\mathrm{H})$, should be designed with $O D=36 \mathrm{~mm}, I D 2=30 \mathrm{~mm}$, $I D=30 \mathrm{~mm}, O H=30 \mathrm{~mm}$ and $I H=8 \mathrm{~mm}$.

Following the same procedure, It was also confirmed that the optimized shape determined by $B_{\text {app }}=3 \mathrm{~T}$ was also effective for the case of $B_{\text {app }}=10 \mathrm{~T}$, and for the case of outer $\mathrm{MgB}_{2}$ TFM cylinder. That is, the optimum shape of the inner lens depends not on the particular bulk materials used and/or the superconducting properties of the outer TFM cylinder, but only the geometry of the magnetic lens.

Figs. 4(a) and 4(b), respectively, show the time step dependence of the magnetic field profile along the $x$-direction across the center of the lens with the optimized design for $B_{\text {app }}=3$ and $10 \mathrm{~T}$. The contour maps of the magnetic field within the magnetic lens at step 5 are also shown in each figure. The $B_{\mathrm{c}}$ value is enhanced up to 6.9 and $15.5 \mathrm{~T}$ at the central region $(-5<x<5)$ at step 5 for $B_{\text {app }}=3$ and $10 \mathrm{~T}$, respectively. The magnetic flux penetration into the $\mathrm{GdBaCuO}$ lens from both the inner and outer peripheries can be observed with increasing applied field, as shown in the inset contour maps. As a result, the shielding effect of the $\mathrm{GdBaCuO}$ lens weakens in external magnetic fields as high as $10 \mathrm{~T}$. During the descending stage (steps 8 and 11 in the figure), the $\mathrm{GdBaCuO}$ TFM cylinder maintains a trapped field similar to $B_{\text {app }}$ at the final step (step 11) as it is magnetized by FCM. The $B_{\mathrm{c}}$ value gradually decreases and settles to final values of $B_{\mathrm{c}}=5.6$ and $12.8 \mathrm{~T}$, respectively, at the center of the lens.

Figs. 4(c) and 4(d), respectively, show the time step dependence of $B_{\mathrm{c}}$ and $B_{\mathrm{c}} / B_{\text {app }}$ at the center of the $\mathrm{GdBaCuO}$ lens for $B_{\text {app }}=3$ and $10 \mathrm{~T}$. In the ascending stage (steps 0-5), $B_{\mathrm{c}} / B_{\text {app }}$ increases linearly and reaches $2.3\left(B_{\mathrm{c}}=6.9 \mathrm{~T}\right)$ for $B_{\text {app }}=3 \mathrm{~T}$. For $B_{\text {app }}=10 \mathrm{~T}$, however, the $B_{\mathrm{c}} / B_{\text {app }}$ vs $T S$ curve gradually bends and the $B_{\mathrm{c}} / B_{\text {app }}$ value at step 5 decreases to 

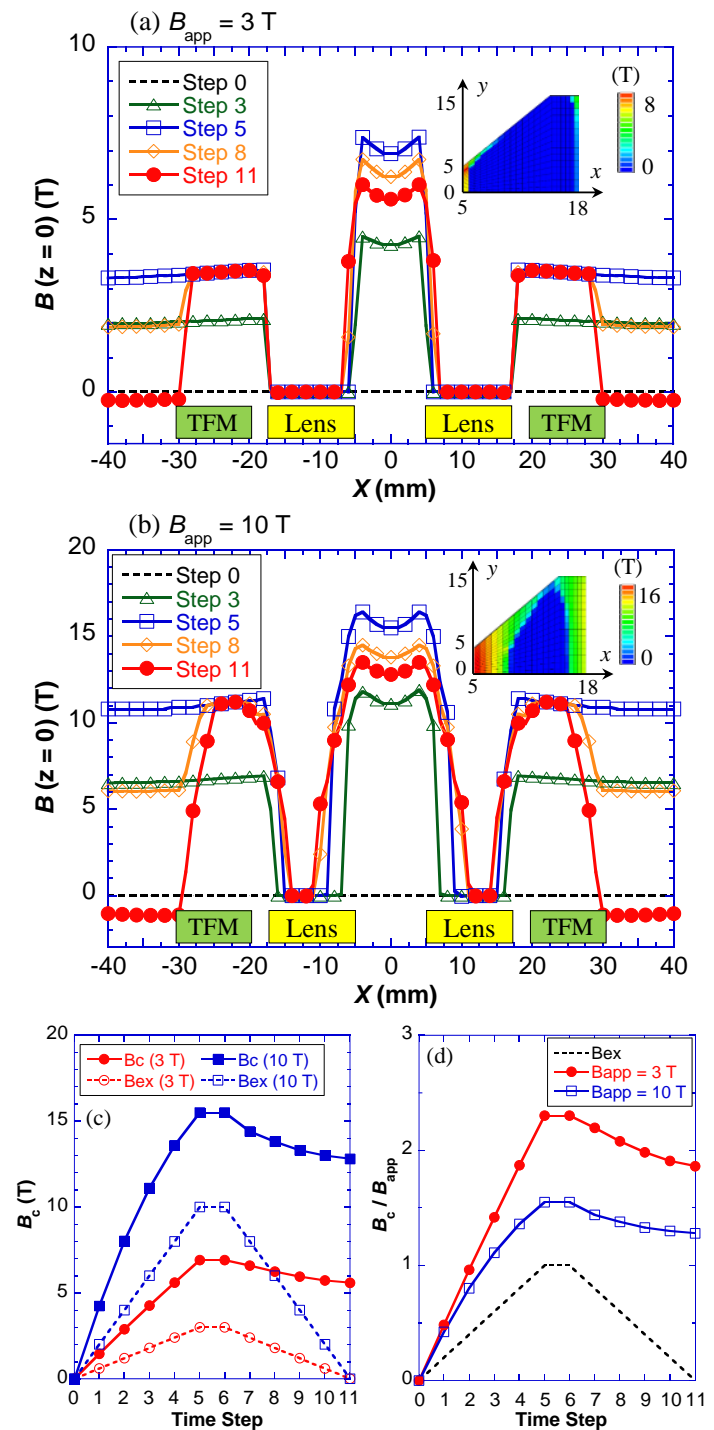

Fig. 4. Time step dependence of the magnetic field, $B$, profiles across the center of the HTFML $(z=0)$ for (a) $B_{\text {app }}=3 \mathrm{~T}$ and (b) $B_{\text {app }}=10 \mathrm{~T}$. Contour maps of the magnetic field profiles at step 5 are also shown in each figure. Time step dependence of (c) the concentrated magnetic field, $B_{\mathrm{c}}$ and (d) the magnetic field amplification factor, $B_{\mathrm{d}} / B_{\text {app }}$ for $B_{\text {app }}=3$ and $10 \mathrm{~T}$.

$1.55\left(B_{\mathrm{c}}=15.5 \mathrm{~T}\right)$ due to the weakened shielding effect of the lens. In the descending stage (steps $6-11$ ), $B_{\mathrm{c}} / B_{\text {app }}$ gradually decreases and final values of 1.86 and 1.28 are achieved for $B_{\text {app }}=3$ and $10 \mathrm{~T}$, respectively.

\section{B. Electromagnetic hoop stress, $\sigma_{\theta}$, during magnetization}

In high-field applications of $\mathrm{REBaCuO}$ bulks, mechanical fracture due to large electromagnetic forces must be avoided. Using the coupled electromagnetic-mechanical model, the hoop stress, $\sigma_{\theta}$, present in the magnetic lens and TFM cylinder in the HTFML was estimated. Figs. 5(a) and 5(b) show the time step dependence of the electromagnetic hoop stress profile, $\sigma_{\theta}$, across the center of the lens $(z=0)$ with the optimized design for $B_{\text {app }}=3$ and $10 \mathrm{~T}$, respectively. During the ascending stage (steps $0-5$ ), a tensile stress exists at the inner periphery of the lens, corresponding to the flux penetration area. The shielding current due to the lens's

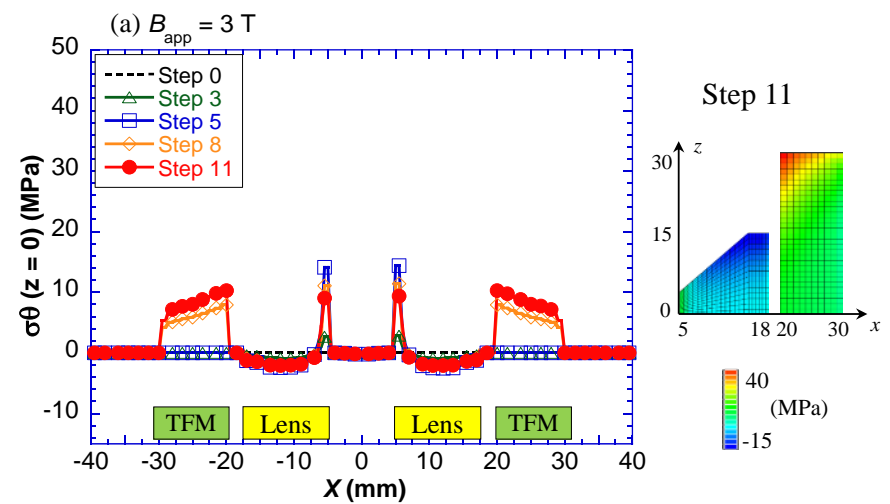

(b) $B_{\text {app }}=10 \mathrm{~T}$

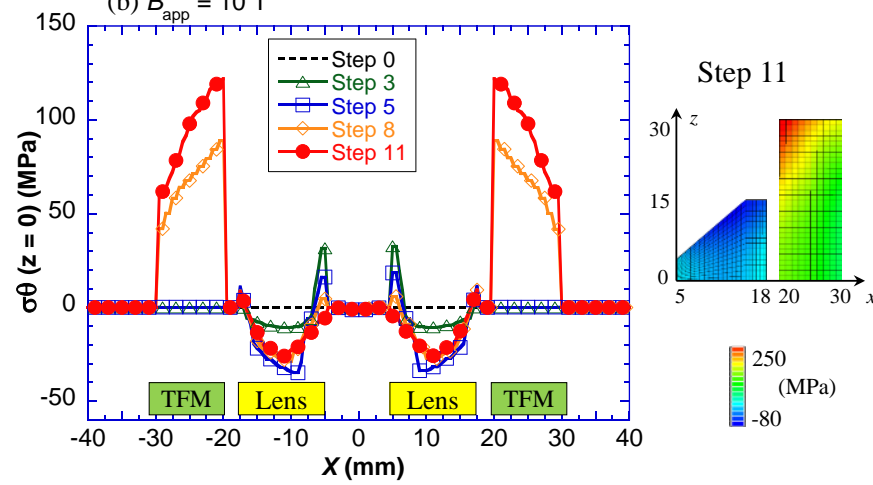

Fig. 5. Time step dependence of the electromagnetic hoop stress profiles, $\sigma_{\theta}$, across the center of the HTFML $(z=0)$ for (a) $B_{\text {app }}=3 \mathrm{~T}$ and (b) $B_{\text {app }}=10 \mathrm{~T}$. The contour map of $\sigma_{\theta}$ at step 11 is also shown in each figure.

diamagnetic property results in a compressive stress in the body of the lens.

On the other hand, during the descending stage (steps 6-11), a large tensile stress in the TFM cylinder, resulting from the Lorentz force, increases with increasing time step. The contour map of $\sigma_{\theta}$ at step 11 is shown in each figure, which details the stress concentration at the inner edge periphery of the TFM cylinder. The maximum $\sigma_{\theta}$ value reaches $+135 \mathrm{MPa}$ at step 11 for $B_{\text {app }}=10 \mathrm{~T}$, which exceeds the fracture strength of typical Ag-doped $\mathrm{REBaCuO}$ bulk materials $(50 \sim 70 \mathrm{MPa})[11,12]$. In the magnetic lens, a maximum tensile stress of $+35 \mathrm{MPa}$ exists at the inner periphery of the magnetic lens and a compressive stress exists in other regions. These results suggest that adequate mechanical reinforcement is necessary, at least for the $\mathrm{GdBaCuO}$ TFM cylinder, but possibly the magnetic lens as well. The demonstration experiment of an HTFML consisting reinforced $\mathrm{GdBaCuO}$ bulks will be conducted on the basis of these simulation results.

\section{CONCLUSION}

In this paper, the optimization of the shape and size of the inner $\mathrm{GdBaCuO}$ magnetic lens in a hybrid trapped field magnet lens (HTFML) was carried out using numerical simulations, which is inserted inside an outer $\mathrm{GdBaCuO}$ TFM cylinder. The mechanical stress in the HTFML with optimized lens was also analyzed during the magnetizing process. The main conclusions are as follows. 
(1) The design optimization of the magnetic lens can be carried out from viewpoints of both the magnetic field amplification and cost-effectiveness.

(2) The HTFML with an optimized lens can achieve concentrated magnetic fields of $B_{\mathrm{c}}=5.6$ and $12.8 \mathrm{~T}$ at the center of the lens for applied magnetic fields of $B_{\text {app }}=3$ and $10 \mathrm{~T}$, respectively.

(3) For $B_{\text {app }}=10 \mathrm{~T}$, a maximum tensile stress of 35 and $135 \mathrm{MPa}$ exist in the magnetic lens and the TFM cylinder, respectively, during the magnetizing process. These results suggest that adequate mechanical reinforcement is needed in a practical design in order to avoid mechanical fracture at high magnetic fields.

\section{REFERENCES}

[1] J. H. Durrell et al., "A trapped field of $17.6 \mathrm{~T}$ in melt-processed, bulk Gd-Ba-Cu-O reinforced with shrink-fit steel," Surpercond. Sci. Technol., vol. 27, no. 8, Aug. 2014, Art. no. 082001.

[2] M. Tomita and M. Murakami, "High-temperature superconductor bulk magnets that can trap magnetic fields of over 17 tesla at 29 K," Nature, vol. 421, pp. 517-520, 2003.

[3] T. Kiyoshi, S. Choi, S. Matsumoto, T. Asano, and D. Uglietti, "Magnetic Flux Concentrator Using Gd-Ba-Cu-O Bulk Superconductors," IEEE Trans. Appl. Supercond., vol. 19, no. 3, pp. 2174-2177, Jun. 2005.

[4] Z. Y. Zhang, S Matsumoto, R Teranishi and T. Kiyoshi, "Magnetic field, temperature and mechanical crack performance of a GdBCO magnetic lens," Supercond. Sci. Technol., vol. 25, 2012, Art. no. 115012.

[5] S. Choi et al., "Magnetic lens effect using Gd-Ba-Cu-O bulk superconductor in very high magnetic field," J. Appl. Phys., vol. 111, 2012, 07E728.

[6] K. Takahashi, H. Fujishiro, and M D Ainslie, "A new concept of a hybrid trapped field magnet lens," Supercond. Sci. Technol., vol. 31, no. 3, 2018, Art. no. 044005.

[7] M. D. Ainslie et al., "Enhanced trapped field performance of bulk hightemperature superconductors using split coil, pulsed field magnetization with an iron yoke," Supercond. Sci. Technol., vol. 29, no. 7, 2016, Art. no. 074003.

[8] M. Jirsa, L. P̊ust, D. Dlouhý and M. R. Koblischka, "Fishtail shape in the magnetic hysteresis loop for superconductors: Interplay between different pinning mechanisms," Phys. Rev. B, vol. 55, pp. 3276-3284, 1997.

[9] T. Kii et al., "Low-temperature operation of a bulk HTSC staggered array undulator," IEEE Trans. Appl. Supercond., vol. 22, no. 3, Jun. 2012, Art. no. 4100904.

[10] H. Fujishiro et al., "Simulation studies of mechanical stresses in $\mathrm{REBaCuO}$ superconducting ring bulks with infinite and finite height reinforced by metal ring during field-cooled magnetization," Supercond. Sci. Technol., vol. 30, 2017, Art. no. 085008.

[11] D. Lee and K. Salama, "Enhancements in current density and mechanical properties of $\mathrm{Y}-\mathrm{Ba}-\mathrm{Cu}-\mathrm{O} / \mathrm{Ag}$ composites," Jpn. J. Appl. Phys., vol. 29, pp. L2017-2019, 1990.

[12] H. Fujimoto and A. Murakami, "Mechanical properties of Gd123 superconducting bulks at 77 K," Supercond. Sci. Technol., vol. 25, no. 5, 2012, Art. no. 054017. 